\title{
Magnetic fields and dynamics of the Sun's interior
}

\author{
Alexander G. Kosovichev \\ W. W. Hansen Experimental Physics Lab, Stanford University, Stanford, CA 94305, USA \\ email:sasha@sun.stanford.edu
}

\begin{abstract}
Advances in helioseismology provide new knowledge about the origin of solar magnetic activity. The key questions addressed by helioseismology are: what is the physical mechanism of the solar dynamo, how deep inside the Sun are the magnetic fields generated, how are they transported to the surface and form sunspots? Direct helioseismic signatures of the internal magnetic fields are weak and difficult to detect. Therefore, most of the information comes from observations of dynamical effects caused by the magnetic fields. I review results of recent helioseismic observations of the magnetohydrodynamics of the solar interior on various scales, including global dynamics associated with the dynamo processes, and formation of sunspots and active regions.
\end{abstract}

Keywords. Sun: activity - Sun: helioseismology - Sun: magnetic fields - Sun: interior - sunspots

\section{Introduction}

Solar and stellar magnetic fields are produced by a dynamo process in the stellar interiors, in which the magnetic field is maintained by turbulent motions against Ohmic dissipation. In astrophysical objects, dynamo can exist when plasma consists of seed magnetic field and flow fields. However, sufficient conditions for dynamo are not welldetermined. Mean-field MHD theories of solar and stellar dynamos predict the cyclic behavior, resembling observed properties of solar cycles such as the butterfly diagram for sunspot formation zone and polar field polarity reversals (Fig. 1). It is well-established from observations that during a solar cycle the zone of bipolar magnetic region emergence migrates from the mid latitudes towards the equator (forming the famous "butterfly" diagram) and that the magnetic flux of the following polarity of the bipolar regions migrates toward the poles causing the polar field reversals. However, our understanding of the underlying physical processes is still schematic.

It is quite clear that despite the long history of observations of solar and stellar activity more systematic and detailed studies, both observational and theoretical, are need to advance our understanding of the dynamo processes. From the observational point of view, it is important to use advances of helioseismology to determine the links between the interior dynamics and surface and coronal phenomena: emergence and evolution of active regions, magnetic flux transport, polar field reversals and magnetic flux dissipation and escape. From the theoretical point, it is necessary to link the dynamo models with observational data, directly related to the magnetic field evolution, such as variations of the differential rotation rate in the form of 'torsional oscillations' and variations of the meridional flow.

One of the puzzling features of solar magnetism is its multi-scale spatial and temporal behavior. High-resolution observations reveal that magnetic field on the Sun's surface is very structured and consists of small, rapidly evolving magnetic elements, the ultimate scale of which is still unresolved. They form active regions and sunspots, which seem to 


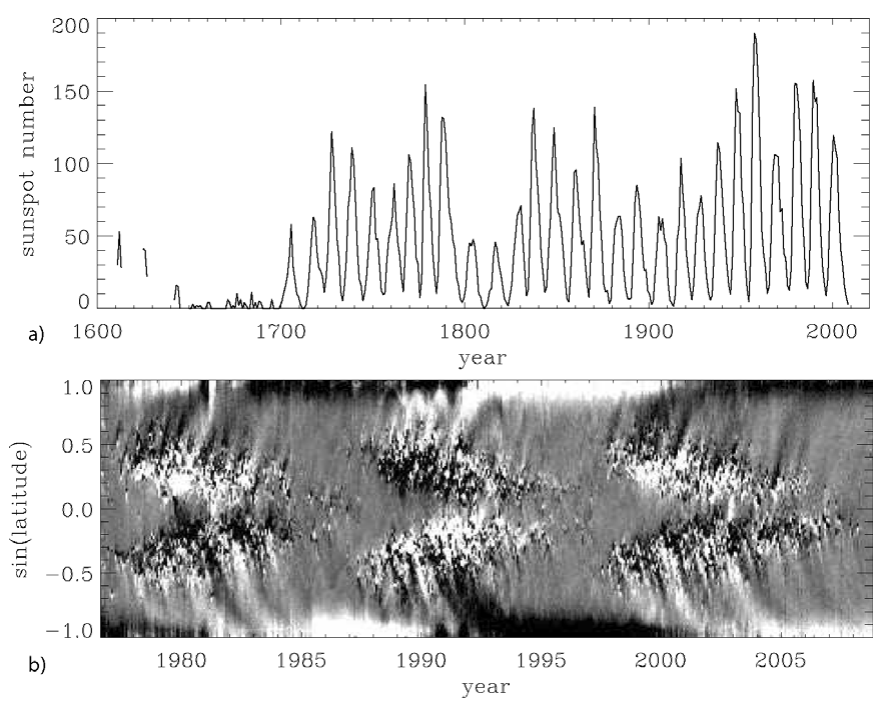

Figure 1. a) The sunspot number record from 1610 to 2008. b) Azimuthally averaged magnetic field as a function of latitude and time for three solar cycles of 1976-2008, observed at the Kitt Peak Observatory. Magnetic field of the positive and negative polarities is shown in white and black colors. Toroidal magnetic field produces bipolar active regions in mid- and low-latitude zones forming the characteristic "butterfly" diagrams. The magnetic field of the following polarity of the active regions is transported to the polar regions causing periodic polarity reversals and forming the poloidal field, which is believed to be a source of toroidal field of future cycles.

emerge randomly. At the same time, solar magnetic fields show a remarkable degree of organization on the global scale, displaying the 'butterfly' diagram and polarity reversals quite regularly with 11-year sunspot cycle (Fig. 1).

Many key questions of solar magnetism remain unanswered. How deep is the dynamo? What is the role of the two radial shear layers: tachocline and subsurface shear layer? What is the relationship between the internal dynamics and magnetic fields? What is the origin of the torsional oscillations? Are there any solar-cycle (11-year) variations in the tachocline? What is the structure of the meridional flows? How do they change with the solar cycle? Why is the solar magnetic field is concentrated in sunspots? How deep are the roots of sunspots? What is the structure and dynamics of the solar plasma beneath sunspots?

\section{Probing solar magnetism and dynamics by helioseismology}

The basic mechanisms of solar and stellar magnetism operate below the visible surface, and thus unaccessible for direct astronomical observations. However, helio- and asteroseismology provides tools for probing the physical conditions inside the Sun and stars. In particular, a great deal of information about the structure and dynamics of the solar interior has been obtained. This unique knowledge about the dynamo process and formation of magnetic structures in the conditions typical for astrophysical plasmas is extremely important for understanding not only the solar magnetic activity but the origin of cosmic magnetism in general.

Helioseismology is based on observations and analysis of solar acoustic waves. These waves are stochastically excited by turbulent convection in a shallow subsurface layer and travel through the solar interior. Methods of helioseismology are generally divided into global, based on analysis on oscillation frequencies of resonant normal modes, and 

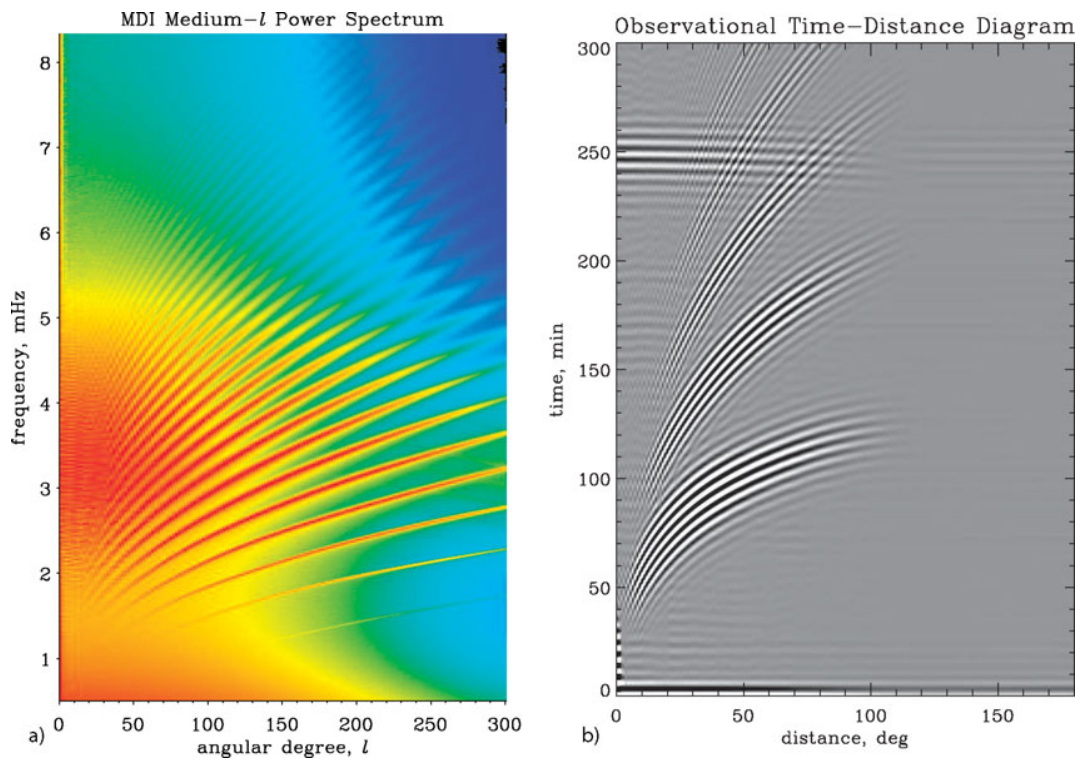

Figure 2. a) Power spectrum of solar oscillations, obtained from SOHO/MDI data. The ridges (yellow and red) correspond to global oscillation modes. The lowest faint ridge is the signal of the surface gravity wave (f-mode). The higher ridges correspond to acoustic waves (p-modes). b) The cross-covariance function of solar oscillations (time-distance diagram). The lowest ridge corresponds to acoustic wave packets traveling directly between two surface points through the interior. The higher ridges correspond to waves arriving after intermediate reflections (bounces) from the surface.

local, based on measurements of properties of acoustic waves (such as travel times and dispersion) in local areas. Figure 2a shows the oscillation power spectrum used for global helioseismology diagnostics. Figure $2 \mathrm{~b}$ shows the time-distance diagram, which is calculated as cross-covariance of the oscillation signals observed at various locations as a function of distance between the measurement points and lag time. This diagram is used to measure travel times of acoustic wave packets traveling through the interior. In magnetic regions these measurements correspond to travel times of fast magneto-acoustic waves. The travel times are used to infer perturbations of the wave speed and flow velocities using a 3D tomographic inversion procedure (Kosovichev 1999). The methods of helioseismology are being extensively developed and tested by the use of numerical simulations.

\section{Solar dynamics and dynamo}

\subsection{Dynamo models}

Most of our current understanding of solar magnetism comes from turbulent dynamo models developed on the principles of mean-field electrodynamics. The dynamo process in these so-called $\alpha-\Omega$ models consists of cyclic transformations between predominantly poloidal and toroidal states of solar magnetic fields (Parker 1955) as illustrated in Fig. 3. The models predict that the toroidal magnetic field, which is the primary source of magnetic active regions and sunspots, is generated from the poloidal component by the Sun's differential rotation ( $\Omega$-effect), and that the poloidal field is produced by a helical turbulence ( $\alpha$-effect). In the $\alpha-\Omega$ dynamo model the equator-ward migration of sunspots is explained in terms of dynamo waves, and the polar-ward flux transport is a result 

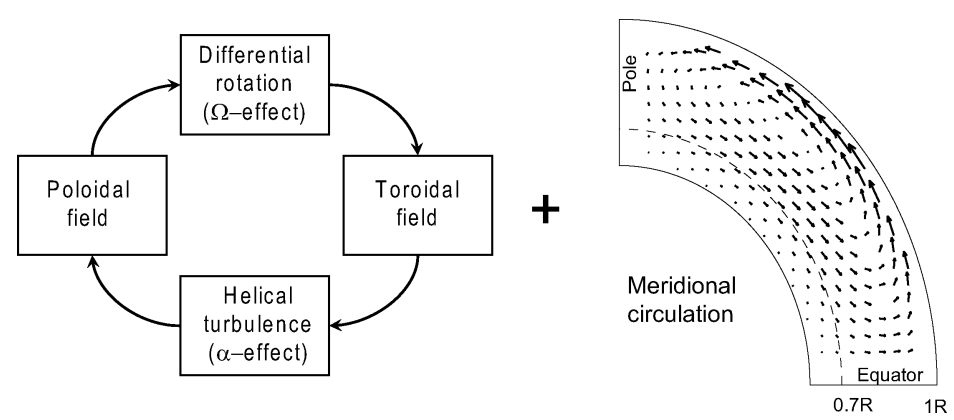

Figure 3. a) A schematic illustration of the Parker's $\alpha-\Omega$ dynamo. b) meridional circulation model. The combination of the $\alpha-\Omega$ dynamo and magnetic flux transport by the meridional circulation gives a flux-transport dynamo (e.g. Dikpati \& Gilman 2008).

of turbulent flux diffusion. To get a better agreement with the observed migration of magnetic flux into the polar regions the models include the flux transport by meridional flows, and are called flux-transport models. For testing the dynamo models it is important to determine the input parameters, such as the differential rotation rate, the structure and speed of the meridional flows, the diffusion and helicity coefficients.

\subsection{Internal differential rotation and zonal flows}

The internal differential rotation is inferred from splitting of frequency multiplets of normal acoustic modes of the Sun. The results of these measurements (Fig. 4a) reveal two radial shear layers at the bottom of the convection zone (so-called tachocline) and in the upper convective boundary layer. A common assumption is that the solar dynamo operates in the tachocline area (interface dynamo) where it is easier to explain storage of magnetic flux than in the upper convection zone because of the flux buoyancy. However, there are theoretical and observational difficulties with this concept. First, the magnetic field in the tachocline must be quite strong, $\sim 60-160 \mathrm{kG}$, to sustain the action of the Coriolis force transporting the emerging flux tubes into high-latitude regions (D'Silva \& Choudhuri 1993). The magnetic energy of such field is above the equipartition level of the turbulent energy. Second, the back-reaction such strong field should suppress turbulent motions affecting the Reynolds stresses. Since these turbulent stresses support the differential rotation one should expect significant changes in the rotation rate in the tachocline. However, no significant variations with the 11-year solar cycle are detected. Third, magnetic fields often tend to emerge in compact regions on the solar surface during long periods lasting several solar rotations. This effect is known as "complexes of activity" or "active longitudes". However, the helioseismology observations show that the rotation rate of the solar tachocline is significantly lower than the surface rotation rate. Thus, magnetic flux emerging from the tachocline should be spread over longitudes (with new flux lagging the previously emerged flux) whether it remains connected to the dynamo region or disconnected. It is well-known that sunspots rotate faster than surrounding plasma. This means that the magnetic field of sunspots is anchored in subsurface layers. Observations show that the rotation rate of magnetic flux matches the internal plasma rotation in the upper shear layer (Fig. 4b) indicating that this layer is playing an important role in the solar dynamo, and causing a shift in the dynamo paradigm (Brandenburg 2005).

Variations in solar rotation clearly related to the 11-year sunspot cycle are observed in the upper convection zone. These are so-called 'torsional oscillations' which represent bands of slower and faster rotation, migrating towards the equator as the solar cycle 

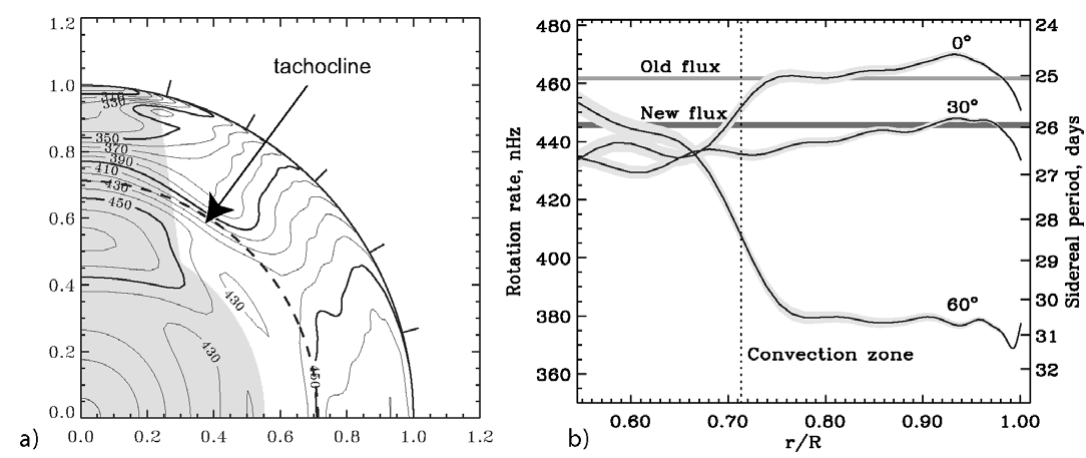

Figure 4. a) Isolines of the rotation rate (in $\mathrm{nHz}$ ) in the solar interior obtained from helioseismology inversion results (Schou et al. 1998). The gray area is where the results are uncertain. Dashed curve shows the location of the bottom of the convection zone and tachocline. b) The rotation rate as a function of radius at three latitudes. The horizontal lines indicate the rotation rate of the surface magnetic flux at the end of solar cycle 22 ("old flux") and at the beginning of cycle 23 ("new flux") (Benevolenskaya, Hoeksema, Kosovichev, \& Scherrer 1999)

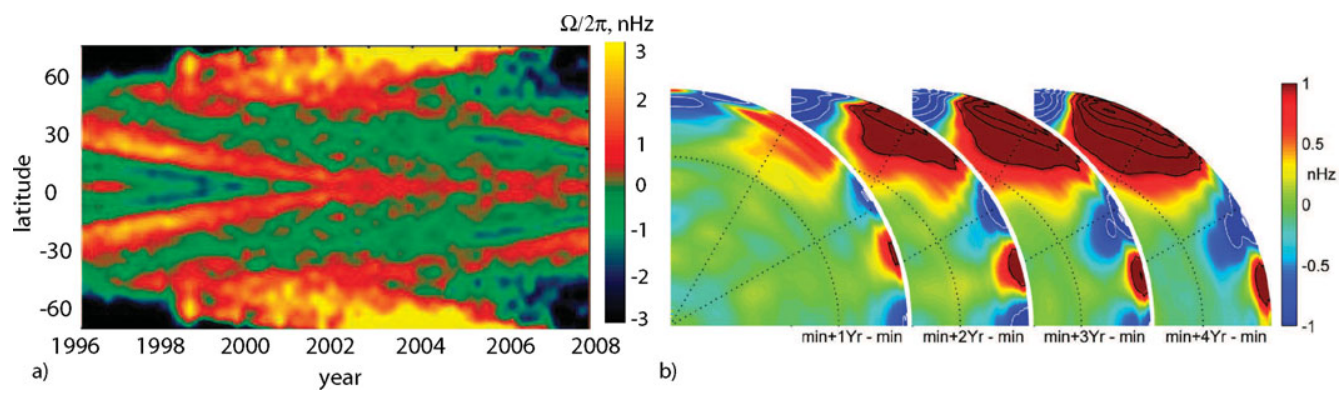

Figure 5. a) Migration of the subsurface zonal flows with latitude during solar cycle 23 from SOHO/MDI data (Howe 2008). Red shows zones of faster rotation, green and blue show slower rotation. b) Variations of the zonal flows with depth and latitude during the first 4 years after the solar minimum. (Vorontsov et al. 2002)

progresses (Fig. 5). The torsional oscillations were first discovered on the Sun's surface (Howard and Labonte 1980), and then were found in the upper convection zone by helioseismology (Kosovichev and Schou 1997; Howe et al. 2000). The depth of these evolving zonal flows is not yet established. However, there are indications that they may be persistent through most of the convection zone, at least, at high latitudes (Vorontsov et al. 2002). The physical mechanism is not understood. Nevertheless, it is clear that these zonal flows are closely related to the internal dynamo mechanism that produces toroidal magnetic field. On the solar surface, this field forms sunspots and active regions which tend to appear in the areas of shear flows at the outer (relative to the equator) part of the faster bands. Thus, the torsional flows are an important key to understanding the solar dynamo, and one of the challenges is to establish their precise depth and detect corresponding variations in the thermodynamic structure of the convection zone. Recent modeling of the torsional oscillations by the Lorentz force feedback on differential rotation showed that the poleward-propagating high-latitude branch of the torsional oscillations can be explained as a response of the coupled differential rotation/meridional flow system to periodic forcing in midlatitudes of either mechanical (Lorentz force) or thermal nature (Rempel 2007). However, the main equatorward-propagating branches cannot be explained by the Lorenz force, but maybe driven by thermal perturbations 

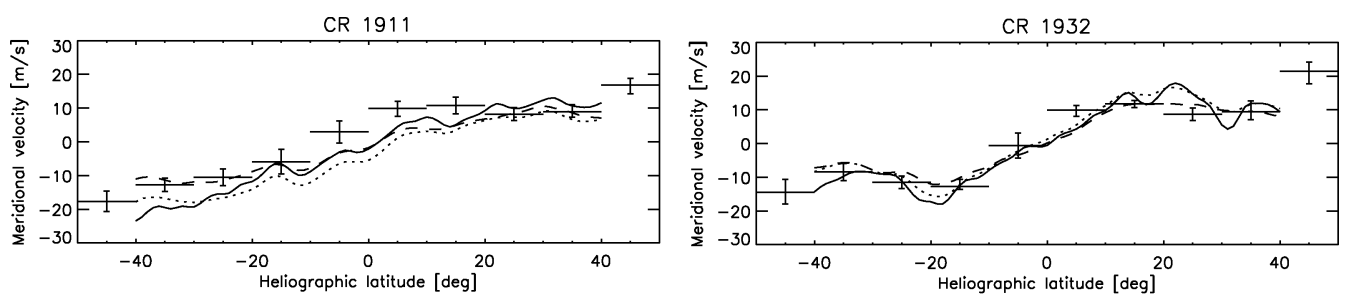

CR 1964

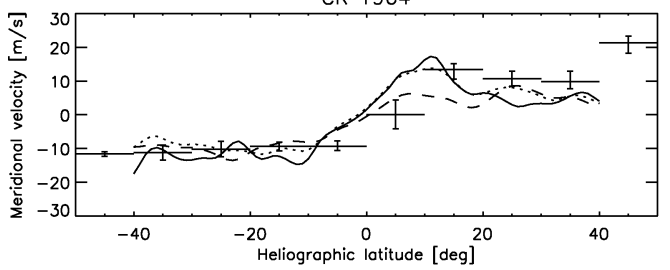

CR 1988

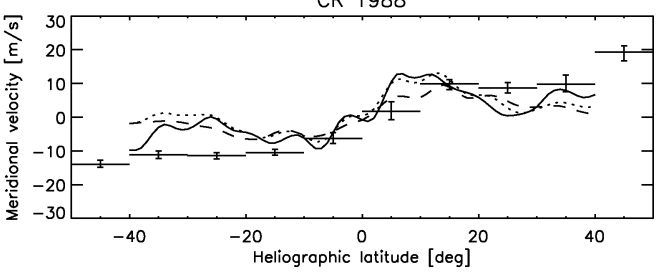

CR 2019
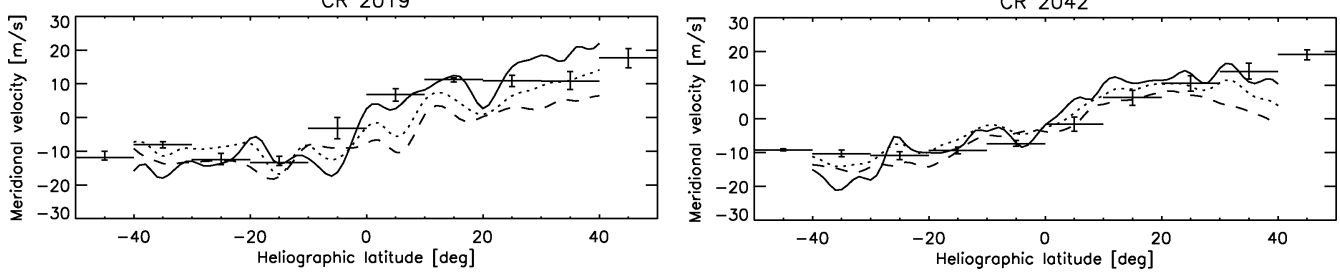

Figure 6. The longitudinally averaged meridional flow speed measured for a set of Carrington rotations by time-distance helioseismology. Solid line plots the time-distance mean meridional flow at 3-4.5 Mm depth, dotted at 6-9 Mm, and dashed at 9-12 Mm. The dots with error-bars represent the 10-degree-bin-averaged values of the flux transport speed derived from the magnetic butterfly diagram (Švanda, Kosovichev, \& Zhao 2007).

caused by magnetic field (Spruit 2003). It is intriguing that starting from 2002, during the solar maximum, the helioseismology observations show new branches of "torsional oscillations" migrating from about $45^{\circ}$ latitude towards the equator (Fig. 5a). They indicate the start of the next solar cycle, number 24, in the interior, and are obviously related to magnetic processes inside the Sun. However, magnetic field of the new cycle appeared on the surface only in 2008.

\subsection{Meridional flows and flux transport}

The meridional flows of the solar plasma have been reliably measured by local helioseismology only in the upper convection zone. These flows are directed from the equator to the polar regions. The return meridional flow expected from mass conservation has not been detected. The helioseismology measurements from SOHO/MDI and GONG show that the speed of the flows significantly varies during the solar cycle. In particular, during the activity maximum the speed decreases. This seems to be explained by the effect of large-scale inflows developed around magnetic active regions (Haber, Hindman, Toomre, \& Thompson 2004; Zhao \& Kosovichev 2004). This decrease may affect the magnetic flux transport in to the polar regions and the polar field reversals. However, the relationship between the meridional flows and the magnetic flux transport is not straightforward (Švanda, Kosovichev, \& Zhao 2007). While, despite a general correspondence there are significant differences between the speeds of the meridional flow flux transport (Fig. 6). There is also an indication that the inflows are predominantly developed around the leading polarity areas of active regions, where magnetic field is stronger and more stable. Helioseismic measurements of the meridional flows are difficult (Giles, Duvall, 

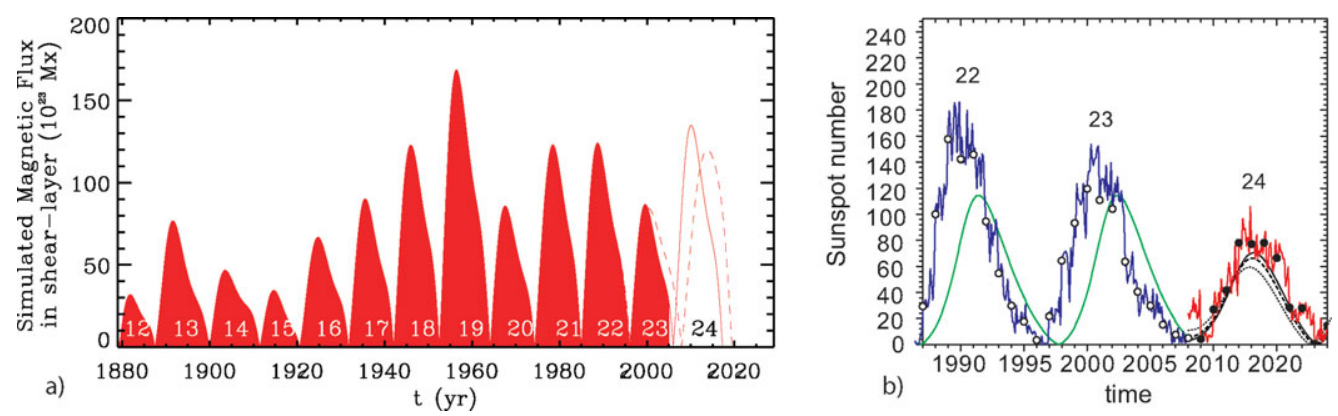

Figure 7. Predictions of solar cycle 24 by using data assimilations and dynamo models: a) flux-transport model (Dikpati, de Toma, \& Gilman 2006), b) model with magnetic helicity balance (Kitiashvili \& Kosovichev 2008).

Scherrer, \& Bogart 1997; Braun \& Fan 1999; Haber et al. 2002; Zhao \& Kosovichev 2004; Krieger, Roth, \& von der Lühe 2007; Mitra-Kraev \& Thompson 2007) because of their low speed, $\sim 10-20 \mathrm{~m} / \mathrm{s}$, but definitely most critical for understanding the solar dynamo mechanism.

\subsection{Predictions of solar cycles}

Because of the insufficient knowledge of the solar dynamics and without a reliable dynamo model physics-based predictions of solar cycles are difficult. Using simple arguments that the new toroidal field (which determines the sunspot magnetic flux) is generated from the poloidal field by differential rotation, and thus the poloidal field strength determines the strength of the sunspot cycle, Schatten (2005) and Svalgaard et al. (2005) predicted that the next solar cycle will be lower than the current one. However, the calculations of Dikpati, de Toma, \& Gilman (2006), based on the calibrated flux-transport dynamo model, suggest that such direct relationship does not hold, and the next cycle will be significantly stronger (Fig. 7a). The recent efforts are to apply data assimilation methods for incorporating observational data in the dynamo models. This approach commonly based on Kalman-filter methods is used, for instance, for weather forecasts. It is proved to be useful for estimating the current state of a system from a set of observations and for predicting future states even when the precise nature of the system is unknown. The data assimilation methods allow to account for the uncertainties in both observations and theory. Kitiashvili \& Kosovichev (2008, 2009a) applied the Ensemble Kalman Filter (EnKF) method to assimilate the sunspot number data (Fig. 1a) into a simple dynamical model of the solar dynamo, which in addition to the standard $\alpha-\Omega$ mechanism considers the evolution of large- and small-scale magnetic helicity (Kitiashvili \& Kosovichev 2009b). The results predict a weak cycle 24 (Fig. $7 \mathrm{~b}$ ), but also indicate that the sunspot number data alone do not provide sufficient constraints, and suggest that synoptic magnetic field data used for further development of this approach along with more detailed dynamo modeling.

\section{Subsurface structure and dynamics of sunspots}

One of the great puzzles of solar magnetism is the concentration of the dynamogenerated magnetic field in compact strong-field structures, sunspots. The sunspots have a fibril structure (Fig. 8a), and detailed observations showed that sunspots represent bundles of magnetic tubes and that the plasma can flow inside sunspots between these bundles (Severny 1965). Following these observations Parker (1979) suggested a cluster 
a)
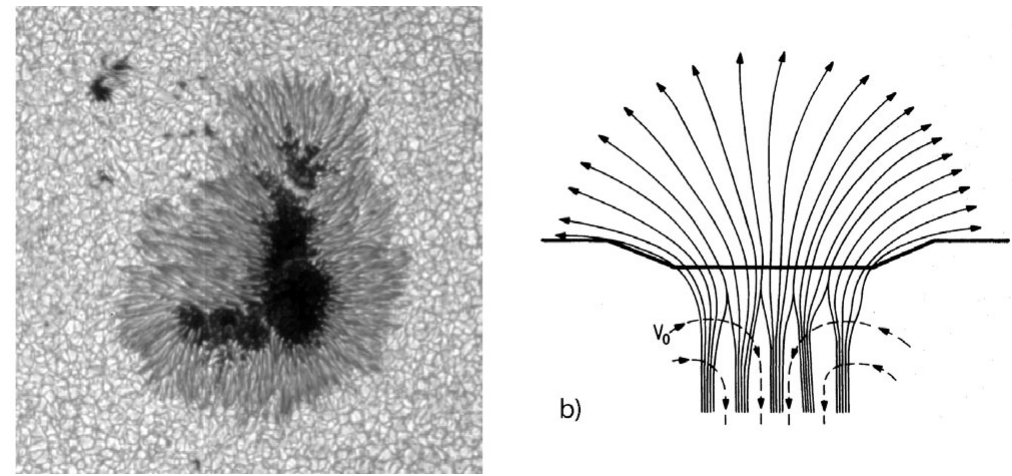

Figure 8. a) High-resolution image of a sunspot from Hinode spacecraft. b) Cluster model of sunspots (Parker 1979).
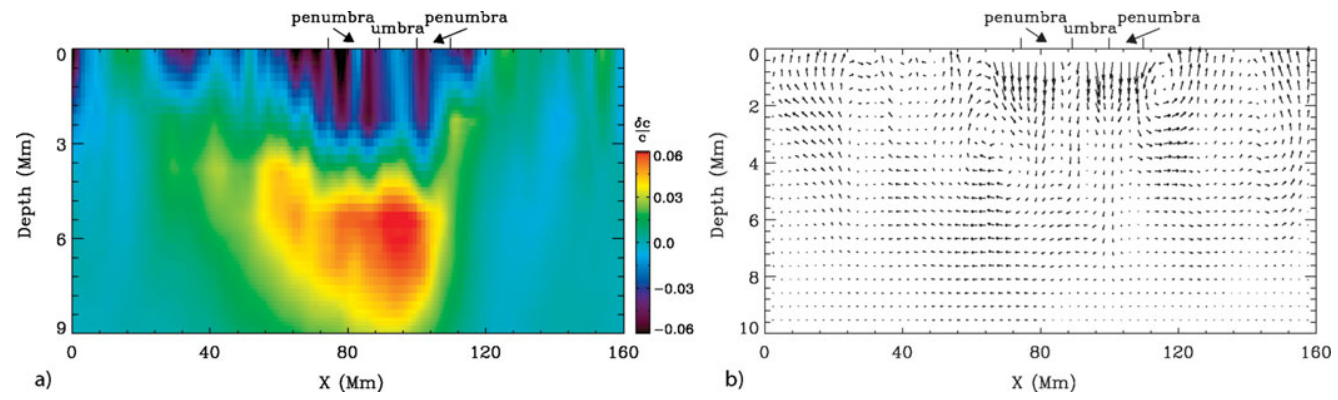

Figure 9. Variations of the acoustic wave speed (a) and the flow field (b) beneath the sunspot shown in Fig. 8a, obtained by time-distance helioseismology from Hinode data (Zhao, Kosovichev, \& Sekii 2009). Red color shows regions where the wave speed is higher than in surrounding plasma, and blue color shows where the wave speed in lower. The longest arrow corresponds to the plasma velocity of $\sim 0.5 \mathrm{~km} / \mathrm{s}$.

model (Fig. 8b), in which the magnetic structure of sunspots is maintained by inflows of plasma. Such inflows can be a part of the large-scale circulation around sunspots originating from the downdraft motions of the cool plasma beneath sunspots. Time-distance helioseismology measurements have shown the existence of the downdrafts and inflows (Duvall et al. 1996; Zhao, Kosovichev, \& Duvall 2001). They also showed that beneath sunspots there is a 4-5 Mm deep region of relatively low wave speed (presumably due to the low plasma temperature), and that in the deeper interior the wave speed becomes higher (Kosovichev, Duvall, \& Scherrer 2000). Recent high-resolution observations from Hinode spacecraft (Kosugi et al. 2007; Tsuneta et al. 2008) have provided a remarkable confirmation of these results and clearly revealed the sunspot cluster structure in the subphotosphere (Zhao, Kosovichev, \& Sekii 2009). Figure 9 shows a vertical structure of the acoustic wave-speed perturbations and flows of the sunspot shown in Fig. 8a. The high-resolution helioseismology observations open new perspectives of studying the sunspot dynamics in much great detail.

\section{Formation and evolution of active regions}

Recent observations and modeling reveal some interesting features of the properties of emerging magnetic flux and associated dynamics on the solar surface and in the upper convection zone. In particular, the local helioseismology results obtained by both, the 


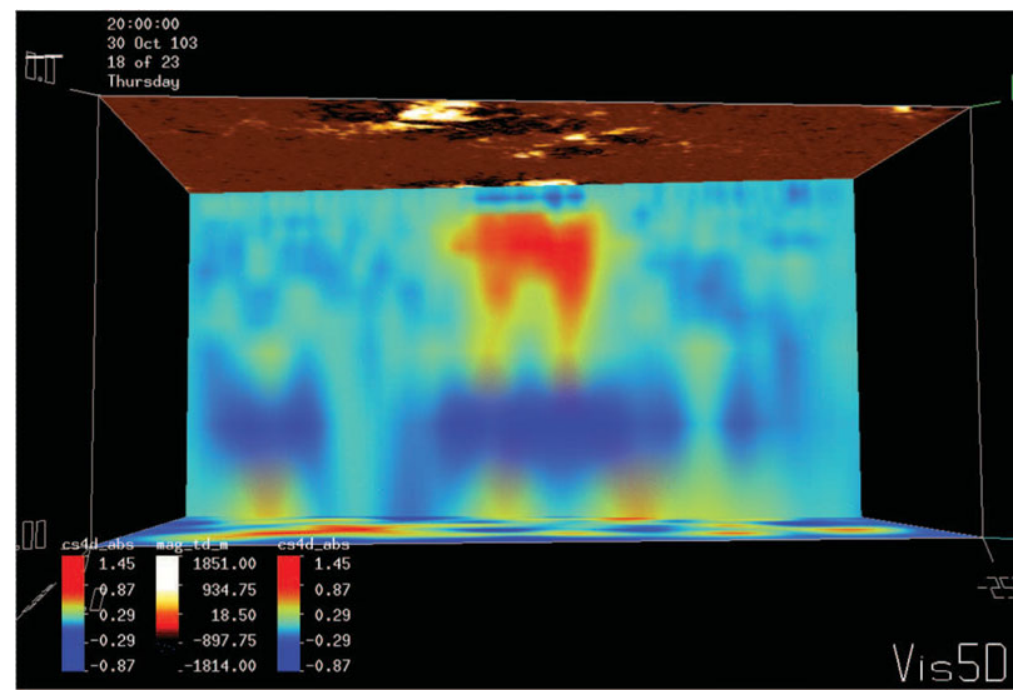

Figure 10. Acoustic wave speed image of active region NOAA 10488 in the solar interior.

(Kosovichev 2009)

time-distance and ring-diagram techniques, show large-scale outflows beneath the surface during most of the emergence phase, and also formation of converging flows around the magnetic structure of sunspots. However, the structure of the vertical flows remains unclear. There is an indication of upflows mixed with downflows at the beginning of emergence, but then the downflows dominate. In the case of AR 10488, there were two or three major flux emergence events. The photospheric magnetic flux rate and subsurface flow divergence show two or three peaks, which are not in phase, but it is unclear if the flux rate precedes the variation of the flow divergence or follows it (Kosovichev 2009).

From the observations it is obvious that the multiple flux emergence events over several days plays important role in the formations and maintaining the magnetic structure of the large active region. This reminds the idea of a common 'nest' in the deep interior (Castenmiller, Zwaan, \& van der Zalm 1986). However, such nests have not been found in the helioseismic images of the subphotospheric magnetosonic wave speed variations, which are currently obtained up to the depth of 40-50 Mm (Fig 10). The wave speed images reveal that the emerging magnetic flux structures travel very fast in the upper convection zone, with a speed of at least $1 \mathrm{~km} / \mathrm{s}$. This makes very difficult the detection of these structures before the magnetic field becomes visible on the surface. However, it should be possible to use the measurements of both, the wave speed variations and flow velocities, for predicting the growth and and decay of active regions and, perhaps, the complexity of their magnetic structure. This task will require a substantial statistical analysis of emerging active regions by methods of local helioseismology.

\section{Conclusion and outlook}

Helioseismology has uncovered intriguing dynamics of plasma in the Sun's interior. Many of the initial results are unexpected and counter-intuitive and are not explained by the current theoretical models. For understanding the basic mechanisms of solar activity, magnetic energy generation, storage and release, it is important to investigate in detail the processes of the dynamics of the tachocline, meridional flows, torsional oscillations, emergence and evolution of active regions, and their relationship to formation 
and evolution of magnetic field structures in the corona, causing magnetic reconnections and plasma eruptions. Helioseismic and Magnetic Imager (HMI) instrument along with the Atmospheric Imaging Assembly (AIA) on Solar Dynamics Observatory scheduled for launch in 2009 will measure Doppler velocity and vector magnetic field, also image hightemperature solar corona, almost uninterruptedly, providing high-resolution ( 0.5 arcsec per pixel) data for these investigations. It is equally important to develop theoretical models of the solar dynamo and sunspot formation, as well as, investigate the magnetic activity on other stars, using long-term monitoring, Doppler imaging techniques and asteroseismology, and understand the place of the Sun in cosmic magnetism.

\section{References}

Benevolenskaya, E. E., Hoeksema, J. T., Kosovichev, A. G., \& Scherrer, P. H. 1999, ApJ 517, L163

Brandenburg, A. 2005, ApJ 625, 539

Braun, D. C. \& Fan, Y. 1999, ApJ 510, L81

Castenmiller, M. J. M., Zwaan, C., \& van der Zalm, E. B. J. 1986, Sol. Phys. 105, 237

Dikpati, M., de Toma, G., \& Gilman, P. A. 2006, Geophys. Res. Lett. 33, 5102

Dikpati, M. \& Gilman, P. A. 2008, J. Astrophys. Aastron. 29, 29

D'Silva, S. \& Choudhuri, A. R. 1993, A\&A 272, 621

Duvall, T. L. J., D’Silva, S., Jefferies, S. M., Harvey, J. W., \& Schou, J. 1996, Nature 379, 235

Giles, P. M., Duvall, T. L., Jr., Scherrer, P. H., \& Bogart, R. S. 1997, Nature 390, 52

González Hernández, I., Kholikov, S., Hill, F., Howe, R., \& Komm, R. 2008, Sol. Phys. 252, 235

Haber, D. A., Hindman, B. W., Toomre, J., \& Thompson, M. J. 2004, Sol. Phys. 220, 371

Haber, D. A., et al. 2002, ApJ 570, 855

Howard, R., Labonte, B. J. 1980, ApJ 239, L33

Howe, R. et al. 2000, Science 287, 2456

Howe, R. 2008, Adv. Sp. Res. 41, 846

Kitiashvili, I., \& Kosovichev, A. G. 2008, ApJ 688, L49

Kitiashvili, I. N., \& Kosovichev, A. G. 2009a, this proceedings

Kitiashvili, I. N., \& Kosovichev, A. G. 2009b, Geophys. Astrophys. Fluid Dyn. 103, 53

Komm, R., Howe, R., Hill, F., \& Morita, S. 2008, ASPC Conf. Ser. 383, 83

Kosovichev, A. G. \& Schou, J., 1997, ApJ 482, L207

Kosovichev, A. G. 1999, J. Comp. Appl. Math. 109, 1

Kosovichev, A. G., Duvall, T. L. Jr., \& Scherrer, P. H. 2000, Sol. Phys. 192, 159

Kosovichev, A. G., 2009, Sp. Sci. Rev., in press

Kosugi, T. et al. 2007, Sol. Phys. 243

Krieger, L., Roth, M., \& von der Lühe, O. 2007, AN 328, 252

Mitra-Kraev, U. \& Thompson, M. J. 2007, AN 328, 1009

Parker, E. N. 1955, ApJ 122, 293

Parker, E. N. 1979, ApJ 230, 905

Rempel, M., 2007, ApJ 655, 651

Schou, J., et al. 1998, ApJ 505, 390

Severny, A. B. 1965, Soviet Astronomy 9, 171

Schatten, K., 2005, Geophys. Res. Lett. 32, 21106

Spruit, H. C. 2003, Sol. Phys. 213, 1

Svalgaard, L., Cliver, E. W., Kamide, Y. 2005, Geophys. Res. Lett. 32, 1104

Švanda, M., Kosovichev, A. G., \& Zhao, J. 2007, ApJ 670, L69

Tsuneta, S. et al. 2008, Sol. Phys. 249, 167

Vorontsov, S. V. et al. 2002, Science 296, 101

Zhao, J., Kosovichev, A. G., \& Duvall, T. L., Jr. 2001, ApJ 557, 384

Zhao, J. \& Kosovichev, A. G. 2004, ApJ 603, 776

Zhao, J., Kosovichev, A. G., \& Sekii, T., 2009, Proc. Hinode-2 conf., ASP Ser., in press 


\section{Discussion}

MAEDER: The circulation you are finding at the convective solar surface is indeed different from the usual meridional circulation, which results from a breakdown of radiative equilibrium in radiative regions. Your circulation velocities are indeed orders of magnitude larger than the "usual" meridional circulation.

Kosovichev: The meridional circulation in the convection zone is produced by the interaction of turbulent convection with rotation. This mechanism is different from the mechanism of the meridional circulation in the radiative zone. Therefore, the mean velocity of the meridional flows in the convection zone is higher than in the radiative zone. Current theoretical models of the meridional flows in the convection zone show that these flows are highly dynamic, and often consist of multiple cells. The multiple cell structure has not been detected by helioseismology but if it exists it will have important implications for the flux-transport dynamo models. Observational and theoretical studies of meridional flows on the Sun are of primary importance for our understanding of solar dynamo.

De Gouveia dal Pino: Comment: There is a poster (P.26) with my PhD student Gustavo Guerrero where we have performed mean field dynamo simulations where we found that the turbulent pumping is dominant over the overall deep meridional circulation to provide the right flux transport to the right latitudes and right depths. Also, if combined with near surface shear, it also provides the appropriate butterfly diagram without requiring a deep meridional flow (Guerrero \& de Gouveia dal Pino, A \& A, 2008).

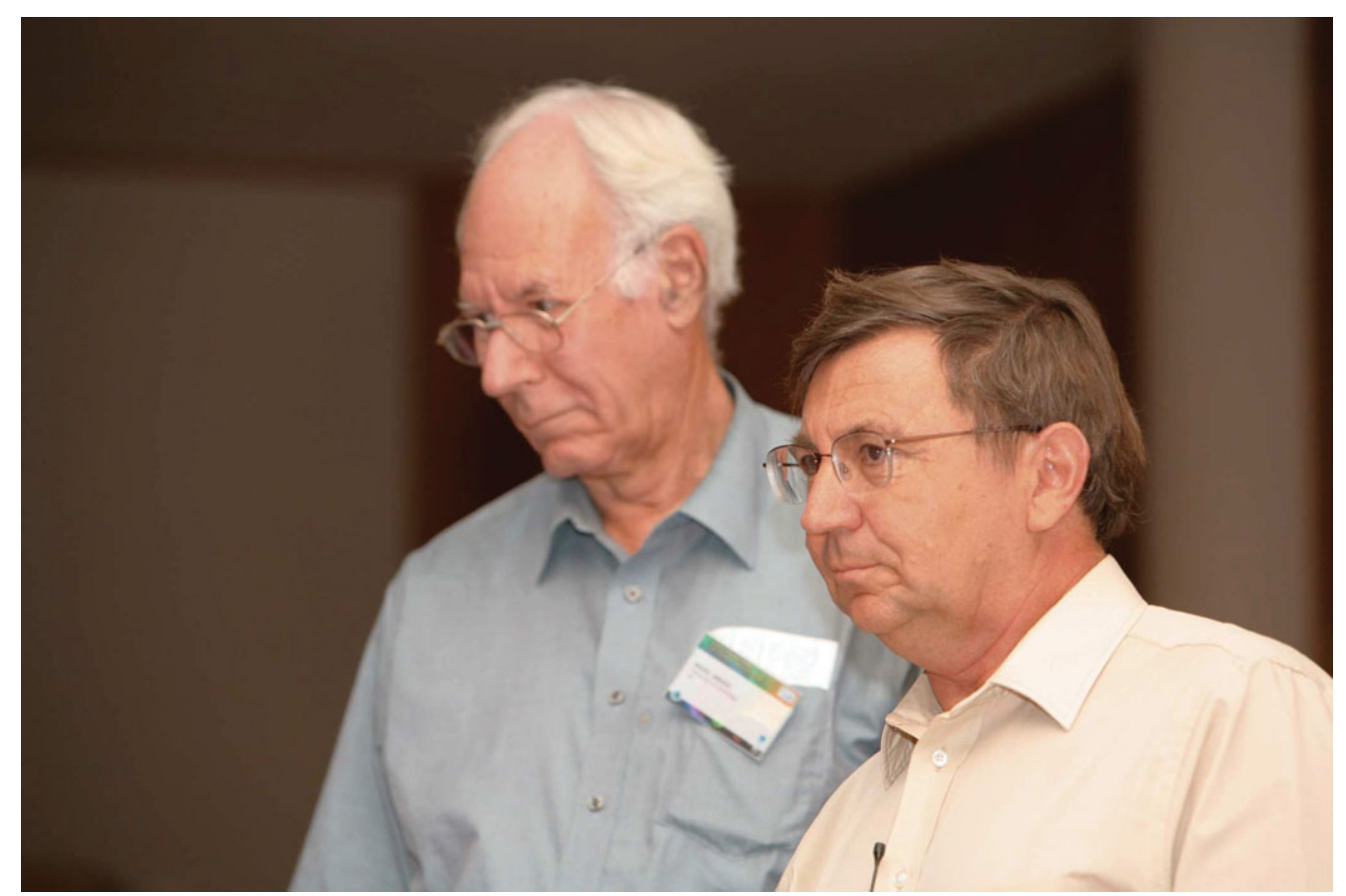

The chairman, left, Nigel Weiss, and the speaker, right, Alexander Kosovichev, eagerly expecting questions 


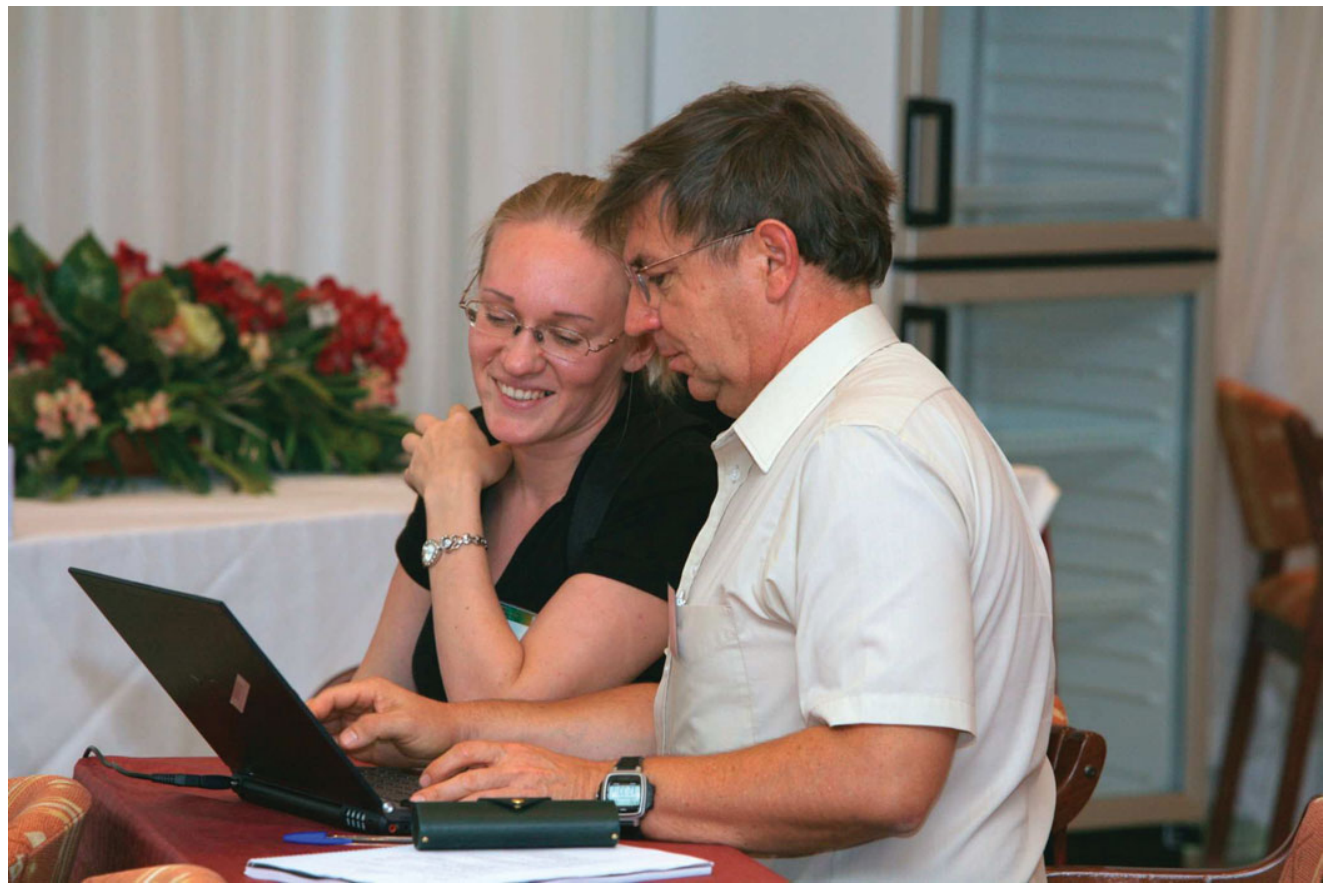

More detailed answering. A. Kosovichev and Irina Kitiashvili.

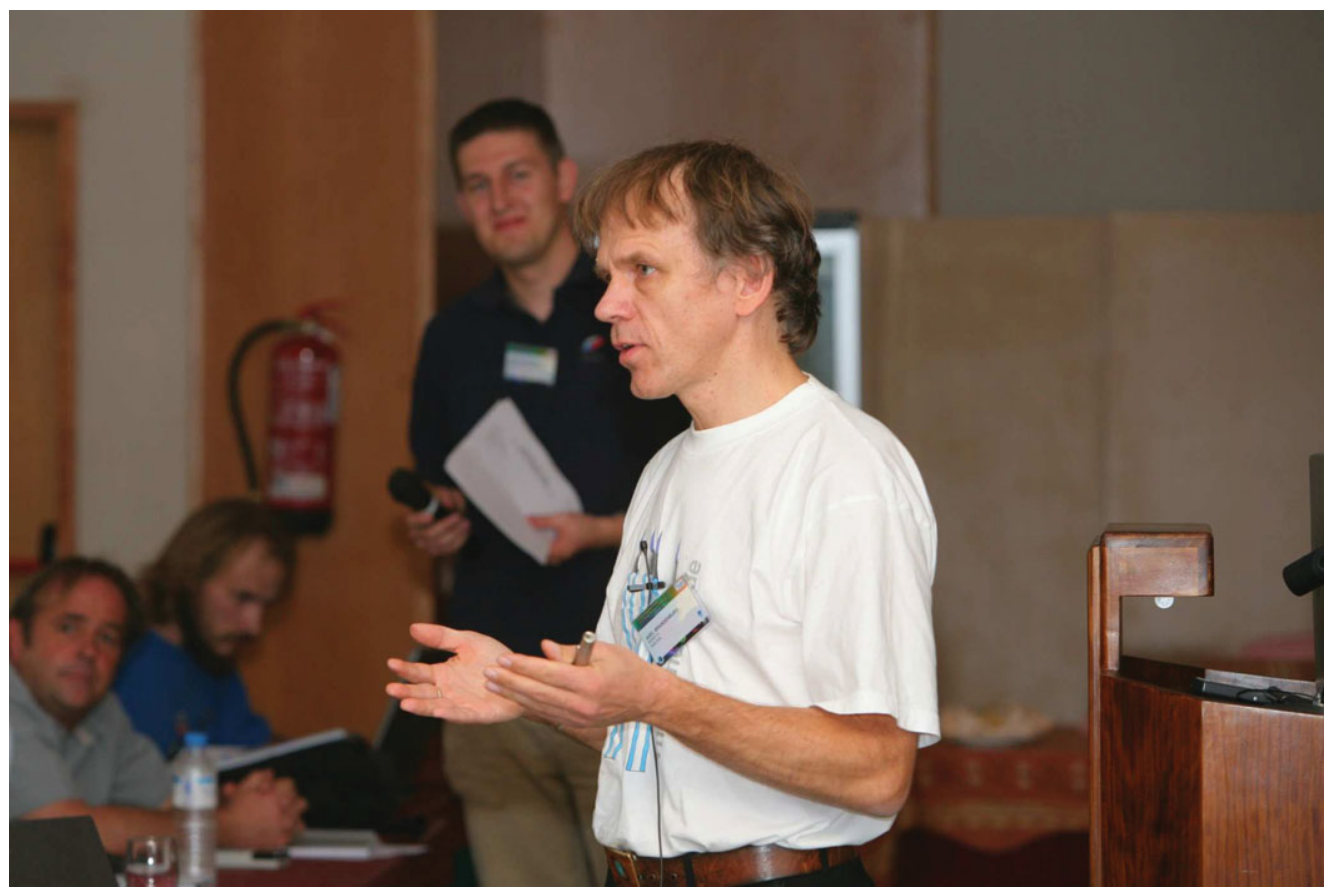

Axel Brandenburg 